\title{
Thermo-mechanical properties of palm fiber plastic (PFP) composites
}

\begin{abstract}
The oil palm Empty Fruit Bunch fibers (size in the range from 75 to $400 \mu \mathrm{m}$ ) have been prepared by wet and dry grinding methods. The prepared fibers were blended with polypropylene to achieve EFB-Polypropylene plastic composite denoted as EFB-PP plastic composite. Various weight contents $(20,35$ and $50 \%)$ of the fibers were reinforced in the polypropylene matrix. The effect of the fiber weight contents on thermal and mechanical properties of the obtained EFB-PP plastic composite was investigated. The wet disk milled fiber reinforced EFB-plastic composite shows the superior mechanical properties.
\end{abstract}

Keyword: Biocomposite; Mechanical property; Natural fiber; Oil palm; Wet disk mill 